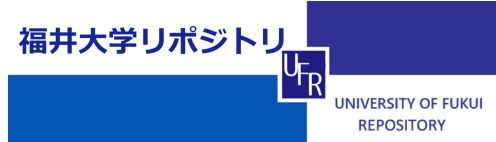

Phase transi ti on i $n$ d- di mensi onal I ong- $r$ ange i nt eracting systems

\begin{tabular}{|l|l|}
\hline 著者 & TATEKAMA Takayuki \\
\hline $\begin{array}{l}\text { j our nal or } \\
\text { publ i cat } \mathrm{i} \text { on } \mathrm{t} \text { i t l e }\end{array}$ & Comput er physi cs communi cat i ons \\
\hline vol une & 117 \\
\hline page $\mathrm{r}$ ange & 190 \\
\hline year & $2007-02$ \\
\hline URL & ht t p: //hdl . handl e. net /10098/6448 \\
\hline
\end{tabular}




\title{
Phase transition in d-dimensional long-range interacting systems
}

\author{
Takayuki Tatekawa ${ }^{\mathrm{a}}$ * \\ ${ }^{a}$ The Center for Continuing Professional Development, Kogakuin University, Tokyo, Japan
}

We present new iterative method for the derivation one-point distribution function in the equilibrium state. For derivation of the distribution function, we must solve Lane-Emden equation. In general case, we solve the equation with iterative method. However the traditional method is not ensured convergence of the algorithm, we cannot often obtain solutions.

In order to obtain the stable stationary distribution function, we apply an iterative method, inspired by a previous one used in $2 \mathrm{D}$ turbulence. Our method ensures entropy increase and convergence of the algorithm. Furthermore, our method can obtain the distribution function quickly [1].

Here we present the phase transition in longrange interacting systems. The Hamiltonian Mean-Field (HMF) model describes the motion of globally coupled particles on a 1D circle. Nevertheless the interaction is described only one cosine function, both the dynamical and the thermodynamical properties of this mode are quite various and complicated. For the HMF model, the extension for 2D model had been proposed [3].

We have extended the HMF model for 3D and $4 \mathrm{D}$ models. Then we analyze the phase-transition for $d$-dimensional models. The Hamiltonian of the HMF models is written as

$$
\begin{aligned}
H & =\sum_{i=1}^{N} \frac{\mathbf{p}_{i}^{2}}{2}+V \\
V & =\frac{1}{2 N}\left[2^{d}-\prod_{a=1}^{d} \sum_{i, j}^{N}\left(1+\cos x_{i j}^{(a)}\right)\right],
\end{aligned}
$$

where $N$ is the number of particles. $d$ is the spacial dimension.

\footnotetext{
*The authors thank the Supercomputer Center, Institute for Solid State Physics, University of Tokyo for the use of the facilities.
}

Table 1

The relation between spacial dimension, order of phase transition, critical energy, and existence of negative specific heat in microcanonical ensemble.

\begin{tabular}{l|ccc}
\hline \hline spacial dim. & $\begin{array}{c}\text { phase } \\
\text { transition }\end{array}$ & $U_{c}$ & $\begin{array}{c}\text { negative } \\
\text { specific heat }\end{array}$ \\
\hline 1 & 2nd & 0.75 & No \\
2 & 2nd & 2.00 & Yes \\
3 & 1 st & 4.28 & Yes \\
4 & 1st & 8.66 & Yes \\
\hline
\end{tabular}

Table 2

The relation between spacial dimension, critical energy, critical temperature, and change of concavity in canonical ensemble.

\begin{tabular}{l|cccc}
\hline \hline spacial dim. & $U_{\text {low }}$ & $U_{\text {high }}$ & $T_{c}$ & $U_{\text {top }}$ \\
\hline 2 & 1.6160 & 2.0413 & 0.54137 & 1.795 \\
3 & 2.7430 & 4.5620 & 0.70811 & 3.517 \\
4 & 4.852 & 9.5409 & 1.0206 & 6.836 \\
\hline
\end{tabular}

We take thermodynamical limit $(N \rightarrow \infty)$ and analyse the equilibrium state in both microcanonical and canonical ensemble. The results for microcanonical and canonical ensemble are shown in Tables 1 and 2, respectively.

\section{REFERENCES}

1. T. Tatekawa, F. Bouchet, T. Dauxois, and S. Ruffo, Phys. Rev. E71 (2005) 056111.

2. M. Antoni and S. Ruffo, Phys. Rev. E52 (1995) 2361.

3. M. Antoni and A. Torcini, Phys. Rev. E57 (1998) R6233. 